\title{
FUTURE OF RESEARCH IN PHARMACEUTICAL ANALYSIS
}

Dear Reader,

I would like to share with you my passion for Quality in Pharmaceutical Analysis

If you think about it, there is no department in a pharmaceutical unit, which is independent from the results of testing in the analytical laboratory. To name a few: New product development, the warehouse, manufacturing department, supply chain, In-process validation and the Quality unit that finally releases a batch, hence involving both middle and top management.

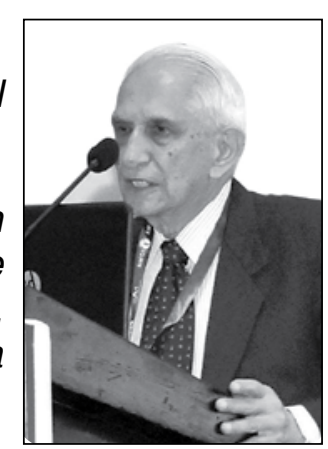

The importance of the correctness of timely results obtained becomes obvious from the above. Hence, it is important to discuss how this can be achieved? Further, data integrity of the activities of the analytical laboratory has become a hot topic in recent years during and after any regulatory Inspection of a unit.

I would like to touch upon two topics of my personal interest here:

1. INDIAN PHARMACOPOEIA (IP) Updates

2. INTERNATIONAL SOCIETY OF PHARMACEUTICAL ENGINEERING (ISPE) Good Practice Guide-TECHNOLOGY TRANSFER.

\section{IP UPDATES}

As we are all aware, the Indian Pharmacopoeia is a book of minimum standards, specifications, limits and methods, with which a drug sample must comply; and it is mandatory for all the drugs manufactured and marketed in India. From time to time these standards are updated and new monographs are added. It is regretful that the stakeholders do not keep updated with the drafts which are published on the website "ipc.gov.in" and become aware only when these are published. By this time, any objections or responses are very difficult for IP commission to deal with and make any changes which may be genuine. Timely and early responses will help in incorporating practical observations in the proposed amendments to monographs or new monographs.

The analyst in a laboratory can contribute a lot by suggestions which improve the test procedures which could save time. With new instrumental techniques a lot is possible. Use of more efficient columns, detectors in chromatography, mass spectroscopy, namely ICMS and LCMS, offer an opportunity for meaningful research. Development at initial R\&D stages, validations and verifications, of test methods as stability-indicating with suitable stress tests will save many of the valid Out Of Specifications- Trends and Unexpected test results and would be of an immense benefit in terms of the time spent in Investigations. "Indian Drugs" publishes many research papers in this discipline of Pharmaceutical Analysis, but these are mainly from academic institutes. Participation in this activity by Industry, which is the most affected party, is minimal. This needs to be improved by better contributions from QC/QA departments of Industry.

\section{ISPE TRANSFER OF KNOWLEDGE FOR ANALYTICAL PROCEDURES}

Often, inspite of SOPs and technology transfer documents, the continued use of the transferred method at the Receiving unit is not satisfactory. This may be due to lack of proper validations for the intended use with different instruments, different reagents and different analysts involved. This many times results in Analyst Error as the Root Cause in the investigation. To minimise these incidents a lot of care needs to be taken at the transfer stage. A lot of detailed discussions and 
suggestions are given in the ISPE Good Practice Guide on Technology Transfer. Persons of appropriate training both at the Sending unit and at the Receiving unit are a must. Many times due to paucity of time shortcuts are done which later on are very difficult and are much more time consuming to identify during any necessary investigation of unexpected results. In the procedures, as much as possible, for example in addition to mixing technique and time, filtration details are worthwhile mentioning. Sometimes these are taken for granted as common sense, which is not as common as we may like to believe!

What is mentioned above in brief is not research but I would like to call it search, for which plenty of material is so easily available in this age of information technology. INDIAN DRUGS publishes papers and articles of original research.

For research in Pharmaceutical Analysis, my thinking is as follows - Massive automation is on the horizon approaching at a fast pace. While I believe robots will not completely replace the QC human pharmaceutical analysts in the laboratory, research is the order of the day in every discipline. More softwares are available and LIMS is being utilized in many laboratories. This should be the future in research in laboratory activities. There are a number of time consuming activities which can be supported. Any such activities which will minimise human errors and save time will definitely be supported by automation in times to come. Time is becoming a very expensive commodity. The pharmaceutical analyst is under tremendous pressure on a daily basis to complete the work allotted to him, without any compromise to quality. Al (Artificial Intelligence) and robotic support will enhance the efficiency of routine analytic procedures in the $Q C$ and QA departments.

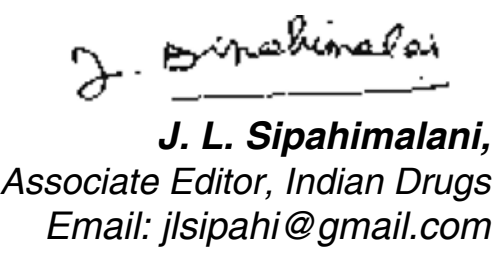

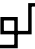

\section{About The Guest Editor}

Shri Sipahimalani is a B.Sc. (Chemistry), B. Pharm Hons. London University, MRSC. Subsequently he worked in Quality Control Department of University College Hospital in London and Pharmacology Department of BDH Ltd in UK for a number of years. He returned to India in 1961. Since then Shri Sipahimalani has been deeply involved in testing and analysis of Pharmaceuticals in Testing Laboratories.

Shri Sipahimalani was selected as a fellow of Royal Pharmaceutical Society of Great Britain in 1979 for distinction in pharmaceutical sciences. He is an Associate Editor of Indian Drugs and IDMA Bulletin and Editor of APA Forum. He has been Chairman of the Technical \& Quality Management Subcommittee of IDMA continuously until 2016 since about last 20 years. Association of Pharmaceutical Analysts (APA) under IDMA was started by him in 1997. He has also been the Convenor of the annual IDMA Pharmaceutical Analysts' Convention since its inception in 1997. The IDMA annual Quality Excellence Awards scheme was initiated and maintained by him since 1985 . He had been actively involved in and contributed to the preparation of IP 1985, IP 1996, 2007, 2010, 2014 and subsequent amendments thereof. He was also the Chairman of IDMA's scientific Committee for Indian Herbal pharmacopoeia. In recognition of his varied achievements, he was honoured with Eminent Analyst Award by IDMA in 2001. Shri Sipahimalani is Chairman of a committee of Experts of IPC, a Board member of ISPE India Affliate and part of IDMA Quality Management and Technical Committee.

$\square$ 